\title{
Die Acidität des Mageninhalts im Säuglings- und Kindesalter bei Milch- und fleischhaltiger Probenahrung.
}

Von

E. J. Huenekens (Minneapolis).

(Aus dem Waisenhaus und Kinderasyl der Stadt Berlin [Oberarzt:

Prof. Finkelstein].)

(Eingegangen am 30. Mai 1914.)

Uber die Frage, ob und von wann ab kleinen Kindern Fleisch gegeben werden soll oder gegeben werden darf, finden sich in der Literatur verschiedene Angaben. Worauf sich der jeweilige Standpunkt der Autoren gründet, ist nicht zu ersehen, da klinische und experimentelle Beobachtungen nicht mitgeteilt worden sind. Bei dieser Sachlage schien es uns wünschenswert, zunächst einmal das Verhalten des Magens im Säuglings. und frühen Kindesalter bei fleischhaltiger Probenahrung zu studieren, und wir berichten in folgendem über die einschlägigen Versuche.

Nach den Untersuchungen von Allarial), Davidsohn') und Salg $\mathrm{e}^{3}$ ) ist die Acidität im Mageninhalt des Säuglings bei Milchnahrung schwach sauer, entsprechend einer Wasserstoffionenkonzentration von etwa $1 \times 10^{-5}$ und ungeeignet, das peptische Ferment zu aktivieren, da letzteres eine viel höhere Acidität - nämlich optimal etwa $1 \times 10^{-2}$ $=1000 \times 10^{-5}$ Normalwasserstoffionen - benötigt. Hieraus ergibt sich, daß wir zur Beantwortung der aufgeworfenen Frage lediglich zu prüfen haben, ob, unter welchen Bedingungen und zu welchem Zeitpunkte beim Säugling resp. beim jungen Kinde die zur Pepsinaktivierung geeignete Acidität zu beobachten ist. Wir wollen dabei

1) Allaria, G. B., Jahrb. f. Kinderheilk. 67, Erg.-Heft, S. 123. 1913.

2) Davidsohn, H., Diese Zeitschr. 2, S. 420. 1911; 4, S. 208. 1912; 9, S. 470.1913.

3) Salge, B., Diese Zeitschr. 4, S. 171. 1912. 
als untere Grenze, bei der eine erhebliche Pepsinverdauung eintreten kann, die Wasserstoffionenkonzentration von $1 \times 10^{-3}=100 \times 10^{-5}$ gelten lassen. Da peptisches Ferment auch beim jungen Säugling nachgewiesen ist, dürfte bei Vorhandensein der genannten Acidität nichts gegen die Annahme, daß eine peptische Fleischverdauung im Magen stattfindet, einzuwenden sein.

Für unsere Untersuchungen haben wir uns der von Davidsohn geübten und ausführlich beschriebenen ${ }^{1}$ ) Anordnung bedient, auf die wir deshalb hier nicht näher einzugehen brauchen. Wir haben fünf Kinder im Alter von $9 \%$ Monaten bis zu 5 Jahren untersucht und haben die betreffenden Ergebnisse in den Tabellen 1-6 ibbersichtlich zusammengestellt. Bezüglich der Nahrung ist das Nötige gleichfalls in den Tabellen angegeben, es sei nur noch bemerkt, daß das Fleisch fein zerschnitten und unter guter Vermischung mit der Milch resp. mit Gemüse und Suppe gereicht wurde. Die Suppe entsprach einer dünnen Fleischbouillon, das Gemüse war, wo nichts anderes bemerkt ist, ohne Milch in der gewönlichen Weise zubereitet und fein püriert.

Tabelle I.

Broesett, $9^{1}$, Monate.

\begin{tabular}{|c|c|c|c|c|}
\hline $\begin{array}{l}\text { Vers.- } \\
\text { Nr. }\end{array}$ & & $\begin{array}{c}\text { Ausge- } \\
\text { hebert } \\
\text { nach } \\
\text { stunden }\end{array}$ & $\begin{array}{c}\text { C. C. } \\
\text { ausge. } \\
\text { hebert }\end{array}$ & $\begin{array}{c}\text { Acidität } \\
=\text { Normalität an } \\
\text { Wasserstoffionen } \\
\times 10^{-5}\end{array}$ \\
\hline 1 & $200^{2} / 3 \mathrm{~L}\left(\mathrm{I}=\right.$ Milch mit $\overline{5}_{0}$, Nährzucker $)$ & 2 & & 1,00 \\
\hline 2 & $200^{2 / 3} \mathrm{~L}$ & 2 & & 3,39 \\
\hline 3 & $200^{2 / 3} \mathrm{~L}$ & 2 & & 0,56 \\
\hline 4 & $2002 / 3 \mathrm{~L}$ & 2 & & 5,62 \\
\hline 5 & $2002 / 3 \mathrm{~L}+10 \mathrm{~g}$ Fleisch & 2 & 50 & 2,34 \\
\hline 6 & $2002 / 3 L+10 \mathrm{~g}$ Fleisch & 2 & 40 & 4,47 \\
\hline 7 & $200^{2 / 3} \mathrm{~L}+10 \mathrm{~g}$ Fleiseh & 2 & 40 & 2,82 \\
\hline 8 & $2002 / \mathrm{a} \mathrm{L}+10 \mathrm{~g}$ Fleisch & 2 & 40 & 2,19 \\
\hline 9 & $200 \% \mathrm{~L}+10 y$ Fleiseh & $11 / 2$ & 30 & 4,68 \\
\hline 10 & 150 Suppe + Gentise in Milch & 1 & 30 & 0,93 \\
\hline 11 & 150 Suppe + Genüse & $11 / 4$ & 75 & 174 \\
\hline 12 & 120 Suppe + Genüse & $11 / 2$ & 30 & 61,7 \\
\hline 13 & 130 Snppe + Genüse & $11 / 4$ & 35 & 87,1 \\
\hline 14 & 150 Suppe + Gemüse & $11 / 2$ & 30 & 32,4 \\
\hline 15 & 150 Suppe $+10 g$ Fleisch + Gemüse & $1^{1 / 4}$ & 30 & 3,24 \\
\hline 16 & 150 suppe $+10 \mathrm{~g}$ Fleisch + Gemüse & 1 & 40 & 1,41 \\
\hline 17 & 150 Suppe $+10 \mathrm{~g}$ Fleisch + Gemüse & 1 & 25 & 7,14 \\
\hline
\end{tabular}

1) Davidsohn, Diese Zeitschr. 9, l. c. 
und Kindesalter bei Milch- und fleischhaltiger Probenahrung.

Tabelle II.

Ema Giersch, 13 Monate.

\begin{tabular}{|c|c|c|c|c|}
\hline $\begin{array}{l}\text { Vers. } \\
\text { Nr. }\end{array}$ & & $\begin{array}{c}\text { Ausge- } \\
\text { hebert } \\
\text { nach } \\
\text { stunden }\end{array}$ & $\begin{array}{c}\text { C. C. } \\
\text { ausge- } \\
\text { hebert }\end{array}$ & $\begin{array}{c}\text { Aciditat } \\
=\text { Normal- } \\
\text { wasserstoffionen } \\
\times 10^{-5}\end{array}$ \\
\hline 1 & 200 Vollmilch mit Brot & $1^{1}$ & 40 & 1,15 \\
\hline 2 & 200 Vollmileh mit Brot & $1^{1}$ & 45 & 0,80 \\
\hline 3 & 200 Suppe mit $10 \mathrm{~g}$ Fleiseh & 1 & 70 & 1,66 \\
\hline 4 & 250 Suppe mit $10 \mathrm{~g}$ Fleisch $+10 \mathrm{~g}$ Spinat & 1 & 50 & 95,5 \\
\hline 5 & 200 Suppe $+10 \mathrm{~g}$ Kartoffel $+10 \mathrm{~g}$ Fleisch & 1 & 40 & 1,23 \\
\hline 6 & 200 Suppe $+10 \mathrm{~g}$ Kohl $+10 \mathrm{~g}$ Fleisch & $1^{1}$ & 35 & 24,6 \\
\hline 7 & 150 Suppe $+10 \mathrm{~g} \mathrm{Kohl}+10 \mathrm{~g}$ Fleisch & 1 & 40 & 13,2 \\
\hline 8 & 200 Suppe $+20 \mathrm{~g}$ Kartoffel $+10 \mathrm{~g}$ Fleisch & $1^{1}$ & 45 & 42,7 \\
\hline
\end{tabular}

Tabelle III.

Max Giese, 17 Monat.

\begin{tabular}{|c|c|c|c|c|}
\hline $\begin{array}{l}\text { Vers. } \\
\text { Nr. }\end{array}$ & & $\begin{array}{c}\text { Ausge- } \\
\text { hebert } \\
\text { nach } \\
\text { Stunden }\end{array}$ & $\begin{array}{c}\text { C. C. } \\
\text { ausge- } \\
\text { hebert }\end{array}$ & $\begin{array}{c}\text { Acidität } \\
=\text { Normal- } \\
\text { wasserstoffionen } \\
\times 10-5\end{array}$ \\
\hline 1 & 200 Vollmilch & 1 & 40 & 2,88 \\
\hline 2 & 200 Vollmileh & $1^{1:}$ & 35 & 3,80 \\
\hline 3 & 200 Vollmileh $+10 \mathrm{~g}$ Fleisch & 1 & 35 & 16,2 \\
\hline 4 & 200 Vollmilch $+10 \mathrm{~g}$ Fleiseh & 1 & 30 & 1,42 \\
\hline 5 & $\begin{array}{l}200 \text { Suppe }+20 \mathrm{~g} \text { Fleisch }+10 \mathrm{~g} \text { Mohr- } \\
\text { rüben }\end{array}$ & $1^{1}$ & 50 & 14,8 \\
\hline 6 & 200 Suppe $+10 \mathrm{~g}$ Gemüse & 1 & 45 & 13,2 \\
\hline 7 & 200 Suppe $+10 \ldots$ Fleisch + Apfelreis & $1^{1}$ & 25 & 148 \\
\hline 8 & 250 Suppe +10 g Fleisch + Kohl & 1 & 40 & 1070 \\
\hline 9 & 250 Suppe $+10 \mathrm{~g}$ Fleisch + Mohrüben & 1 & 40 & 575 \\
\hline 10 & 200 Suppe $+10 \mathrm{~g}$ Fleiseh + Makkaroni & 1 & 50 & 55 \\
\hline 11 & 250 Suppe $+10 \mathrm{~g}$ Fleisch + Spinat & 1 & 40 & 851 \\
\hline 12 & 250 Suppe +10 g Fleisch + grüne Bohnen & 1 & 30 & 148 \\
\hline
\end{tabular}

Tabelle IV.

Georg Koniell, 20 Monate.

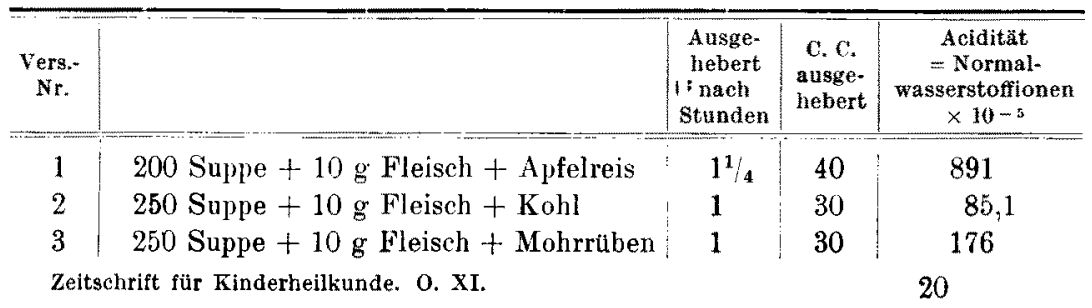


Tabelle V.

Herbert Krieg, 5 Jahre.

\begin{tabular}{|c|c|c|c|c|}
\hline $\begin{array}{c}\text { Yers - } \\
\text { Nr. }\end{array}$ & & $\begin{array}{c}\text { Ausge- } \\
\text { hebert } \\
\text { nach } \\
\text { stunden }\end{array}$ & $\begin{array}{l}\text { C. C. } \\
\text { ausge- } \\
\text { hebert }\end{array}$ & $\begin{array}{c}\text { Acidität } \\
=\text { Normal- } \\
\text { wasserstoffionen } \\
\times 10^{-5}\end{array}$ \\
\hline 1 & 200 Tollmilch + Brot & 1 & 30 & 2,00 \\
\hline 2 & 200 Vollmileh + Brot & 1 & 35 & 2,19 \\
\hline 3 & 250 Suppe $+10 y$ Flcisch + Nohrüben & 1 & 45 & 141 \\
\hline 4 & 250 Suppe +20 gleisch + Bohmen & 1 & 40 & 1410 \\
\hline 5 & 250 Suppe +20 g Eleisch + Kartoffeln & 1 & 30 & 69,2 \\
\hline 6 & 250 Suppe $+20 g$ Fleisch + Kartoffeln & 1 & 45 & 1820 \\
\hline
\end{tabular}

Tabelle VI.

Durchschnittswerte.

\begin{tabular}{|c|c|c|}
\hline & Probenahrung & 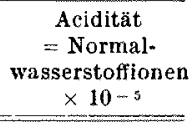 \\
\hline Broesett, $9^{1}$. Monate & $\begin{array}{l}\text { Milch } \\
\text { Fleisch und Gemüse }\end{array}$ & $\begin{array}{l}2,80 \\
3,96\end{array}$ \\
\hline Ema Giersch, 13 Monate & $\begin{array}{l}\text { Milch } \\
\text { Fleisch and Gemüse }\end{array}$ & $\begin{array}{l}0,98 \\
29,8\end{array}$ \\
\hline Max Giese, 17 Monate & $\begin{array}{c}\text { Mileh } \\
\text { Fleisch und Gemüse }\end{array}$ & $\begin{array}{l}8,10 \\
409\end{array}$ \\
\hline Georg Komoll, 20 Monate & Fleisch and Gemüse & 367 \\
\hline Herbert Krieg, 5 Jahre & $\begin{array}{c}\text { Milch } \\
\text { Fleisch und Gemüse }\end{array}$ & $\begin{array}{l}1,71 \\
860^{-71}\end{array}$ \\
\hline
\end{tabular}

Aus den vorstehenden Tabellen ergibt sich zunächst, daß bei allen untersuchten Kindern, selbst bei dem fünfjährigen, die Acidität im Mageninhalt nach Milchprobefrühstück stets schwach sauer ist, gut übereinstimmend mit dem vorher für den künstlich genährten Säugling angegebenen Wert. Die Tatsache, daß der Mageninhalt des Sauglings die erwähnte schwachsaure Acidität bei Milchnahrung aufweist, kann also nicht zu dem Schlusse verwertet werden, daß die Salzsäureproduktion des Säuglingsmagens der Ausdruck einer noch unfertigen Funktion ist; sie beruht vielmehr vermutlich darauf, daß dic Milch cinen besonders schwachen Reiz für die Magensekretion darstellt und ein großes Säurebindungsvermögen besitzt, worauf Aron ${ }^{1}$ ) erst kürzlich wieder hingewiesen hat. Andrerseits soll nun

') Aron, H., Jahrb. f. Kinderheilk. 79, S. 288.1914. 
aber nicht behauptet werden, daß die Salzsäureproduktion der Magenschleimhaut gleich nach der Geburt ebenso wie während des späteren Lebens funktioniert; wir werden nachher unsere Ansschauungen über diese Funktion noch etwas präsizieren. In guter Ubbereinstimmung mit den vorangehenden Utberlegungen zeigt Tabelle I weiterhin, daß bei einer aus Suppe und Gemüse bestehenden Probenahrung (vgl. Versuch Nr. 11-14) die Acidität im Mageninhalt des $91 / 2$ Monate alten Säuglings bedeutend höher ist als nach einfachem Milchprobefrühstück, und daß die Gegenwart einer kleinen Menge Milch (vgl. Versuch Nr. 10) schon genügt, um die Acidität beträchtlich herunterzudrücken. Demgemäß haben wir auch späterhin vorgezogen, den Kindern das Fləisch nicht in Milch, sondern gemeinsam mit Suppe und Gemüse zu verabfolgen.

Aus Tabelle I ergibt sich schließlich noch, daB der betreffende Säugling bei einer aus Suppe, Gemüse und Fleisch bestehenden Probenahrung eine schwächere Acidität im Mageninhalt aufweist als nach Suppe und Gemüse allein, und zwar ist die Säuerung so schwach, daß eine nennenswerte Pepsinverdauung nicht stattfinden kann. Hier überwiegt also die säurebindende Fähigkeit des Fleisches gegenüber ihrer sekretionserregenden.

Die Untersuchungen, welche an einem 13 Monate alten Kinde in ganz entsprechender Weise angestellt und in Tabelle II protokolliert sind, ergeben etwas höhere Aciditätswerte des Mageninhalts nach fleischhaltiger Probenahrung, ohne indessen den zur Aktivierung postulierten Schwellenwert zu erreichen. Einen derartig hohen Aciditätsgrad finden wir dagegen bei den drei übrigen Kindern, welche im Alter von 17 resp. 20 Monaten und 5 Jahren stehen. Wir müssen also unter Zugrundelegung der bei unseren Versuchskindern erlangten Resultate zu dem Schlusse kommen, daß erst im Laufe des zweiten Lebensjahres eine peptische Verdauung des gereichten Fleisches im Magen sich abzuspielen scheint. ${ }^{1}$ )

Es erhebt sich nun die Frage, ob obiges Resultat zu dem Schlusse verwertet werden kann, daß die Salzsäuresekretion der Magenschleimhaut eine Funktion ist, die erst in der zweiten Hälfte des zweiten Lobensjahres ihre volle Höhe erreicht. Unseres Erachtens

1) Anmerkung: Die Aciditätswerte bei fleischhaltiger Probenabrung zeigen ziemlich große Schwankungen. Da aber trotzdem in einwandfreier Weise ein Ausschlag in dem oben gedeuteten Sinne zu erkennen ist, ist es überflüssig, hier auf die Ursache dieser Schwankungen näher einzugehen. 
ist dieser Schluß nicht bindend; besteht doch die Möglichkeit, daß eine mehr oder weniger frühe Zufütterung fester Nahrung zur Milchkost das Resultat beeinflußt. Es ist nicht unmöglich, daß unsere beiden jüngsten Kinder deshalb auch nach fleischhaltiger Probemahlzeit eine so geringe Acidität im Mageninhalt aufweisen, weil ihre Nahrung immer noch vorwiegend Milch war, die, wie schon erwähnt, einen besonders geringen Reiz auf die Magensekretion ausübt. Die drei älteren Kinder, bei denen sich eine für die Pepsinverdaunng hinreichende Acidität im Mageninhalt ergeben hat, sind jedenfalls schon längere Zeit mit vorwiegend gemischter, teilweise sogar fleischhaltiger Kost (vgl. Kind Krieg, Tabelle V) ernährt worden und die Tatsache, daß die Acidität im Mageninhalt nach Suppe und Gemüse annähernd dem Sehwellenwert der Pepsinverdauung entspricht, könnte zu der Annahme verwertet werden, daß die Ernährung mit gemischter Kost für die Gewöhnung der Kinder an Fleisch vorteilhaft ist. Untersuchungen an Säuglingen, denen schon sehr früh feste Nahrung zugefüttert wird, könnte diese Frage entscheiden.

Mag nun das in der zweiten Hälfte des zweiten Lebensjahres beobachtete Auftreten der zur Pepsinverdauung hinreichenden Acidität eine Funktion des Alters, der vorher gereichten gemischten Kost oder beider Faktoren zusammen sein - da nach vielfältiger klinischer Erfahrung am besten im sechsten Lebensmonat mit der Zufütterung fester Nahrung begonnen wird und auch unsere Versuchskinder so ernährt worden sind, berechtigen unsere Ergebnisse zu der Schlußfolgerung, daß ohne besonderen Grund vor der zweiten Hälfte des zweiten Jahres Fleisch zweckmäßigerweise nicht verabfolgt werden soll.

Wir sind uns selbstverständlich dessen bewußt, daß das Fehlen der peptischen Magenverdauung noch nicht den Schluß zuläßt, daß Fleisch überhaupt nicht im Magendarmkanal verdaut werden kann; es ist allgemein bekannt, daß im Reagensglas hierzu das Trypsin und Erepsin des Darmkanales hinreicht, über das unsere Untersuchungen nichts aussagen. Indessen wir wissen auch, da $B$ die peptische Vorverdauung die tryptische Spaltung erheblich begünstigt (vgl. Fischer und Abderhalden ${ }^{1}$ ), Oppenheimer und Aron $\left.{ }^{2}\right)$ ) und dürfen deshalb wohl annehmen, daß für den empfindlichen Darm des jugendlichen Organismus das Fehlen dieser Vorverdauung höchst bedeutsam ist.

1) Fischer, E., und E. Abderhalden, Zeitschr. f. physiol. Chemie 40, S. 215.1903 .

2) Oppenheimer, C., und H. Aron, Hofm. Beiträge 4, S. 279. 1903. 
Klinische Erfahrungen auf unserer Abteilung haben denn auch gezeigt, daß die Fleischdarreichung bei Säuglingen zu dyspeptischen Erscheinungen führen kann.

\section{Zusammenfassung.}

1. Die Acidität im Mageninhalt der untersuchten, im Alter von $91 / 2$ Monaten bis zu 5 Jahren stehenden Kinder nach Milchnahrung ist übereinstimmend schwach sauer; der Säuregrad entspricht dem unter gleichen Bedingungen für den künstlich genährten Säugling gefundenen, nämlich entsprechend einer $\left[\mathrm{H}^{-}\right]=1 \times 10^{-5}$. Die Ursache dieses Verhaltens ist darin zu suchen, dab die Milch einen geringen Sekretionsreiz ausübt und stark säurebindend ist.

2. Die Acidität im Mageninhalt nach einer aus Suppe und Gemüse bestehenden Probemahlzeit ist höher als nach reiner Milchnahrung. Der hier gefundene Aciditätsgrad entspricht dem Schwellenwert zur Aktivierung der Pepsinverdauung.

3. Nach einer aus Suppe, Gemüse und Fleisch bestehenden Probe. nahrung ist die Acidität im Mageninhalt bei dem 91/2 und 13 Monate alten Kindern unzureichend für eine peptische Fleischverdauung im Magen, zureichend aber bei den drei älteren Kindern im Alter von 17 resp. 20 Monaten und 5 Jahren. Bei jungen Kindern wird deshalb zweckmäBigerweise erst von der zweiten Hälfte des zweiten Jahres mit der Fleischnahrung begonnen.

Berlin, Städtisches Waisenhaus und Kinderasyl. 